upper, and screw on the lower instrument, which was left lax on the thigh at the upper third. During the first and following day, he was unable to bear the pressure of either instrument for a longer period than half an hour to an hour-sometimes less; and experienced considerable pain in the calf of the leg, as well as along the tibia. It was, however, remarked that he bore pain unusually long; and being an intelligent man, he controlled the circulation by the feeling of pulsation in the tumour, which he was sensible of, and which he was cautioned not to obstruct completely.

On the 22 nd, he says he passed a restless night, from pain in the knee; but towards morning he relaxed the instruments, and slept; a slight turgescence of the veins around the knee being only perceptible when pressure was put on. The temperature of the limb is not much lower than the opposite; it has a flannel roller applied. The pressure throughout this day was kept up regularly and alternately with the two instruments, and at no period entirely obstructing the pulse in the tumour, or discontinuing pressure. He had each night, from the 15 th, half a grain of acetate of morphia, and eight drops of tincture of digitalis, as a draught.

23rd. - The tumour is sensibly harder, and a few turns of the screw of the lower instrument easily controls the circulation through it. The pressure from the pad in Scarpa's angle has irritated the integuments, and the lower instrument was moved down, and pressed on the artery in the middle third of the thigh. This enabled him to bear the instrument on a much longer time; and being without sleep, he observed the pressure having regularly and completely controlled the pulse in the tumour during this night.

In examining the limb on the morning of the 24th, I relaxed both instruments, and found the pulsation of the tumour quite ceased; nothing more than a fremitus perceptible. The instruments were kept on the thigh, with but slight pressure, to ensure safety, for the two successive days. The tumour felt hard, and he complained only of stiffness in the knee-joint. The temperature of the limb was somewhat less, but no cedema whatever occurred, nor was there any lividity visible during the whole period of pressure.

26th.-The tumour is perfectly solid, and diminished in size; feels no inconrenience but rigidity of the limb, and his bealth has in no way suffered.

31st.-Is walking about the ward, able to extend the limb, and place his weight on it; surface of the tumour painted with iodine; and gentle friction is relieving the rigidity.

Aug. 13th.-Sill in the hospital; the tumour in the ham is not larger than a Spanish filbert; has perfect good use of the leg; the pulsation of the femoral artery felt to the inferior third of the thigh, likewise in the collateral branches; is in very good health, but the heart's action has been feeble throughout; and upon minute examination of the aorta in the epigastric region, a soufflet is audible, distinct from the heart's action; from which fact it is my intention to invalid him from the service, the fleet at present lying in the Bay.

Naval Hospital, Gibraltar, August, 1852.

\section{GOUT AND TRISMUS FOLLOWING INJURY OF THE FOOT.}

\section{BX J. M. SALTER, Esq., Dorset.}

Mr. $\mathrm{H}-$, a respectable yeoman in the neighbourhood of Blandford, Dorset, met with a severe accident on the 23rd of December last. His left foot was caught in a thrashing-machine, by which the fore-part of his boot was torn away, together with the extremities of three of his toes as far as the first joints. Having removed the portions of crushed bone and lacerated in. tegument, I formed a llap over the stump of the great toe, and dressed the parts in the usual way. There was scarcely any hæmorrhage; the foot was very pale and numbed. His age being more than seventy-three, I of course anticipated a troublesome case, and told him there was danger of tetanus or trismus, although from my past experience at home and abroad I felt sure of heing able to bring him through safely, and preventing the trismus (shonld it occur) from being fatal; but a great deal would depend upon my directions being strictly adhered to. The accident occurred about four P.M. At six P.M. I gave acetate of morphia, a quarter of a grain; calomel, three grains; powder of rhubarb, three grains: divide into two pills, to be taken directly. About nine P.M. I gave the following mixture:-Epsom salts, one ounce; potassio-tartrate of antimony, two grains ; infusion of senna, six ounces and a half; syrup, one ounce; aromatic spirits of ammonia, three drachms: to have three tablespoonfuls every four hours until the bowels are moved. That night the sleep was excellent, a nice moisture on the skin, and the next day he could ot feel that anything had occurred to the foot.

The next night I repeated the pills of morphia, which produced the same sleep and moisture as on the previous night; but the next morning tetanus made its appearance in the extremities, followed by symptoms of trismus. He complained of great lassitude, and considerable pain and difficulty in moving the jaws; the countenance assumed a cadaverous appearance. I gave him muriate of morphia, a quarter of a grain; powder of rhubarb, three grains, in form of a pill, to be taken directly. I then administered a good strong glass of brandy-and-water; an hour after, a dose of cordial mixture, with aromatic spirits of ammonia, and repeated the brandy and-water three or four times in the course of the day, with the mixture as above.

On the fifth day, gout made its appearance in the right foot, and trismus again set in. The morphia-and-rhuburb pills were repeated, with brandy-and-water, as before. The gout left the foot, and attacked the stomach, at the same time the patient complaining of great pain in the jaws. Nitrate of potash, a drachm and a half; wine of colchicum, three drachms; aromatic spirits of ammonia, three drachms; powder of rhubarb, ten grains; syrup, an ounce; add water to six ounces : two tablespoonfuls to be taken every two hours. By the third dose relief was procured, and he felt perfectly free from pain.

During the first three or four days there was a little hæmorrhage, but nothing of importance. There was considerable sloughing, and the foetor was intolerable. On the seventh day symptoms of gangrene, and a white line reaching from the toes to the ankle. Having cleansed it with warm water, $\mathrm{I}$ applied tincture of lytta to the sloughs, and poultices saturated with black-wash. At night repeated the calomel-and-morphia pill. Next day another severe attack of the jaws came on. Repeated the morphia-and-rhubarb pill, with brandy-and-water, and giving the aperient mixture. Slept well. On the ninth day all the symptoms of trismus had disappeared, and he felt his jaws as well as ever; the pulse soft, full, and regular; spirits cheerful; system quiet. Being excited by some unexpected visitors that day, considerable irritation followed, and the trismus appeared again the following day, but was subdued by the repetition of the former remedies, and he slept comfortably that night. On the tenth day he was composed, but had a little delirium, and while sleeping comfortably about two in the morning was seized with tetanus in his arms and legs, which awoke him; it then seized the jaws with greater violence than before. The morphia pills were repeated, with the aromatic spirits of ammonia, two drachms, in a mixture, at intervals, and during that day he took nearly a pint of brandy. The foot assumed a more unhealthy appearance, more gangrenous, and the line to the ankle more apparent; pulse small and feeble; tongue coated and feverish. Gout returned during the day; hicenp and delirium. The morphia pills were repeated three times that day, with the aperient mixture. His foot was held over a foot-pan with water nearly boiling; a large cloth five times doubled was dipped in the water, and applied to the foot; this caused much pain, but was continned for an hour. The patient was easier after, and the hot water was repeated in the evening; and at bed-time, calomel, three grains; powder of rhubarb, three grains; acetate of morphia, a quarter of a grain: make a pill, to be taken immediately. He had some sleep; hiccup diminished; delirium less; tongue and pulse the same as in the day.

January 3rd, 1852.-Was evidently better in every respect, but he complained occasionally of pain and inability to move his jaws, which he did for some time afterwards. Some days he felt as if he had never been affected; on others, very strong tendency to absolute trismus. I continued at intervals the pills, the gout mixture, and the brandy, regulating his bowels with the aperient mixture above. Living near him, I had every opportunity of watching him closely, and attending to every symptom as it occurred; and I had the satisfaction of seeing him walk half a mile by the latter end of the month with ease. Being fond of walking, he continued his exercise, and in March could travel four miles on foot without feeling any inconvenience, and now continues his usual exercise in good health.

Stourpaine, Dorset, Angust, .1852.

\section{CASE OF PLACENTA PRARIA.}

\section{By GEORGE BENNETT, Esq., M.R.C.S. Eng., Sydney.}

During the practice of my profession in Sydney, New South Wales, since 1836 , and having during that period an extensive midwifery practice, I have only met with five cases of placenta prævia; in the whole of these cases I invariably detached the placenta previous to the delivery of the child, considering it con- 